\title{
A review of cathode-arc coupling modeling in GTAW
}

\author{
Alireza Javidi Shirvan $^{1}$ • Isabelle Choquet $^{1}$ (D)
}

Received: 30 September 2015 / Accepted: 19 February 2016

(C) International Institute of Welding 2016

\begin{abstract}
Material properties of welds are strongly influenced by the thermal history, including the thermo-fluid and electromagnetic phenomena in the weld pool and the arc heat source. A necessary condition for arc heat source models to be predictive is to include the plasma column, the cathode, and the cathode layer providing their thermal and electric coupling. Different cathode layer models based on significantly different physical assumptions are being used. This paper summarizes today's state of the art of cathode layer modeling of refractory cathodes used in GTAW at atmospheric pressure. The fundamentals of the cathode layer and its physics are addressed. The main modeling approaches, namely (i) the diffusion approach, (ii) the partial LTE approach, and (iii) the hydrodynamic approach are discussed and compared. The most relevant publications are systematically categorized with regard to the respective physical phenomena addressed. Results and process understanding gained with these models are summarized. Finally, some open questions are underlined.
\end{abstract}

Keywords (IIW Thesaurus) GTA Welding · Cathodes · Mathematical models $\cdot$ Tungsten electrodes $\cdot$ Reviews · Simulating $\cdot$ Plasma $\cdot$ Heat flow

Recommended for publication by Study Group 212 - The Physics of Welding

Isabelle Choquet

isabelle.choquet@hv.se

Alireza Javidi Shirvan

alireza.javidi-shirvan@hv.se

1 Welding group, Dept. Mechanical Engineering, University West, Trollhättan, Sweden

\section{Introduction}

Despite the very long application of electric arc welding in the industry and the almost continuous development of this process, there is still need for deeper process understanding and predictive simulation models. Predictive simulation models should establish the link between the welding process parameters that are controlled in the factory and the resultant material properties of the welded product, such as the microstructure and residual stresses. The material properties of a weld are strongly influenced by the thermal history. Thanks to several decades of research, it is now recognized that the thermal history is closely coupled to the thermofluid and electromagnetic phenomena occurring in both the weld pool and the arc heat source [1]. A necessary condition for arc heat source models to be predictive is to include the plasma column, the cathode, the anode, and their respective coupling. This coupling allows calculation of the temperature and current density distributions on the cathode and anode surfaces rather than prescribing them, e.g., through the extrapolation of spectroscopic measurements in the plasma column. It has a critical influence on the thermal and electrical fields within the arc, and thus on the heat transfer to the base metal.

In electric arcs, the central part of the plasma column can be at local thermal equilibrium (LTE), e.g., for a gas tungsten arc with argon gas. Its colder edges however usually deviate from LTE $[2,3]$. In contrast to the plasma column, the cathode and anode layers always present several severe non-equilibria including deviations from thermal, ionization, and charge equilibrium. These non-equilibria extend over regions that differ in terms of physics, modeling scales, and thicknesses, depending on the combinations of process parameters such as the 
shielding gas (e.g., mono or diatomic for instance), the pressure, or the electric current [4-9].

Cathode and anode layers have been investigated since the early 1900s. Several reviews already exist; the first one was done by Ecker [10]. Among the more recent is the review proposed by Franklin [5] on the plasma sheath boundary region and the evolution over time of its understanding and modeling, by Benilov [4] on the cathode layer modeling, and by Heberlein et al. [8] and Shkol'nik [9] on the anode layer modeling. Anode and cathode layer modeling is important for several domains of applications, e.g., high-intensity discharge (HID) lamps, electric arc welding, arc furnaces, plasma torches, and electrical switches. To our knowledge, the first application of a 2D coupled arc-cathode simulation model including the cathode layer was done by Delalondre [11] for a pure tungsten cathode with a $60^{\circ}$ conical tip, currents of 100, 200, and $300 \mathrm{~A}$ and arc lengths of 10 and $20 \mathrm{~mm}$. These configurations were rather similar to gas tungsten arc welding (GTAW) although the arc length was significantly longer, and the cathode material was pure tungsten rather than a tungsten alloy. The application of a 2D coupled arc-cathode model including the cathode layer for the simulation of a typical GTAW configuration is more recent; e.g., Zhu et al. [12] simulated a 5-mm long arc, coupled with a thoriated tungsten cathode (with typical conical tip) and an electric current of 200 A. Since then, several authors have investigated thermal and electric arc-cathode coupling applied to GTAW. Nevertheless, this problem is still open. There is as yet no consensus in the welding community on the modeling of the arc-cathode coupling. Different models based on significantly different physical assumptions are being used.

The aim of this paper is to summarize today's state of the art of cathode layer modeling of refractory cathodes as used in GTAW at atmospheric pressure, when considering the whole cathode arc system. The paper is divided into three main sections. In Sect. 2, the fundamentals of the cathode layer and its physics are addressed. The main approaches used, namely the diffusion approach, the partial LTE approach, and the hydrodynamic approach are then discussed and compared in Sect. 3. The most relevant publications are systematically categorized with regard to the respective physical phenomena addressed. Some results and process understanding gained with these models are summarized in Sect. 4. Finally, some open questions are underlined in conclusion.

\section{Physics of the cathode layer in GTAW at atmospheric pressure}

The physics of the cathode layer and the electron emission at the cathode surface are tightly coupled. Three important electron emission processes are the thermionic, the thermo-field, and the field emission; see Fig. 1 (right-hand side). Thermionic emission is dominant at high cathode surface temperature. Thermo-field emission is thermionic emission enhanced by the lowering of the surface potential barrier induced by the presence of a space charge layer (or sheath) in front of the cathode. It is associated with low to moderate cathode surface electric field strength. Field emission prevails at high cathode surface electric field strength and low cathode surface temperature.

For refractory material, the cathode melting and vaporization rates are low and in practice usually ignored; field emission is also negligible [13]. For the refractory cathodes used in GTAW applications, the electron emission is usually modeled based on the Richardson-Dushman equation for thermionic emission, supplemented with a correction for the so-called Schottky effect. This effect expresses the influence of the cathode surface electric field on the electron emission [13] and allows extending the thermionic emission model toward thermo-field emission. Other laws were also developed for modeling the electron current density produced by thermofield emission [13, 14]. The domain of validity of the Richardson-Dushman-Schottky thermo-field emission law was investigated for a tungsten cathode by $\mathrm{He}$ [15] and his results were reported by Vacquié [13].

The Richardson-Dushman-Schottky emission model involves two variables, namely the temperature and the electric field strength at the cathode surface (which are among the unknowns of the cathode layer problem), and two dependent parameters. The first parameter $A_{R}=\lambda_{R} A_{o}$ is the Richardson constant, where $A_{o}$ denotes a universal constant while $\lambda_{R}$ is a material-specific factor. The second parameter is the work function $\phi$. It can be defined as the minimum energy that must be given to a valence electron moving freely inside the metal to be liberated from the metal surface. With this minimum energy, the electron has no kinetic energy for moving further

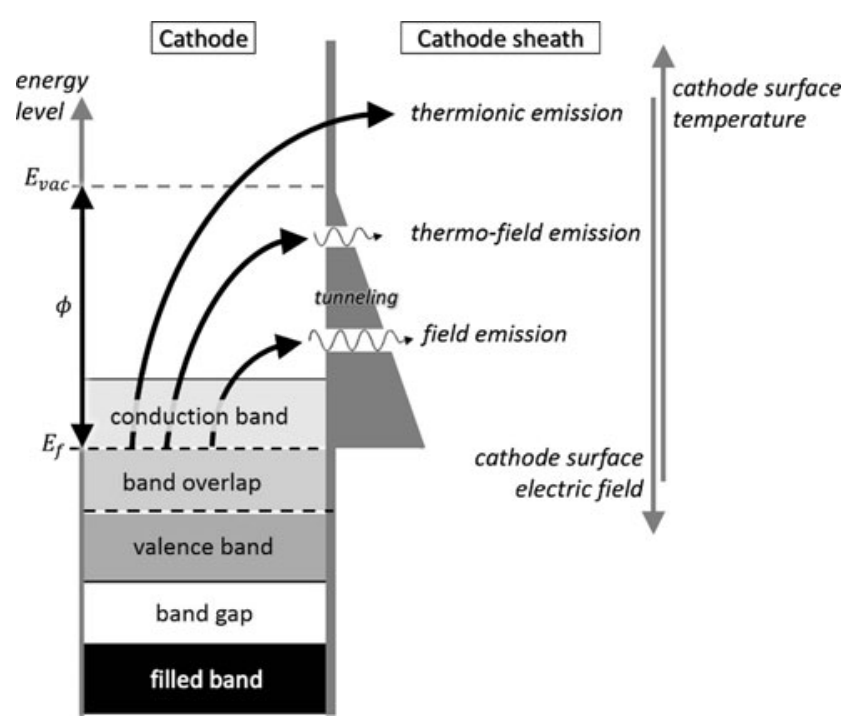

Fig. 1 Schematic energy level diagram of a cathode (left) and main electron emission processes (right) 
away from the surface. Also, in this definition of the work function, the valence electron occupies the highest energy level (when the system is in its ground state), which is the Fermi level of energy $E_{f}$. An electron escaping with a minimum energy causes a negative charge just outside the metal surface. In turn, a positive charge just inside the metal surface is formed. This causes a sheet of dipoles at the metal surface, implying repulsive force acting upon the electrons as they try to escape from the metal surface. This repulsive force at the surface forms a potential barrier of energy called the energy vacuum level $\left(E_{\text {vac }}\right)$. The minimum energy for valence electrons to escape the metal and overcome the surface potential barrier is thus $\phi=E_{v a c}-E_{f}$ (see Fig. 1). This energy is the work function. If, in addition, a layer of positive ions builds up outside the metal surface on the top of the dipole layer, as in the sheath or space charge layer of a cathode layer in GTAW, it facilitates the electron emission by lowering the surface potential barrier.

The set of parameters $A_{R}$ and $\phi$ is generally determined via experimental measurements fitted to an a priori expression of the emission law [16-19]. Several authors studied thoriated tungsten for example and observed that its work function $\phi$ varies with the arc current, and thus with the material temperature. This phenomena was observed at low current (from 2.5 to $12.5 \mathrm{~A}$ for HID lamps [19]) as well as at higher current (100 and $200 \mathrm{~A}$ [16], and from 30 to $200 \mathrm{~A}$ [17], [18] for configurations similar to GTAW). As further explained by Aillero Marín [17] for instance, this variation is due to the temperature dependence of the diffusion, the evaporation, and the re-deposition of thorium atoms. When modeling the cathode-arc coupling, the choice made for $\lambda_{R}$ and $\phi$ is known to have a significant influence on the temperature field calculated in the arc [20].

Another emission process taking place at the cathode surface is the secondary electron emission induced by highly energetic ions as they collide with the cathode. A review on the models and data for modeling this emission process in the presence of an argon gas was performed by Phelps and Petrović [21]. These authors underline that the secondary electron emission depends on both the material and the conditions at the metal surface (e.g., impurities, oxidation). They also observed that for thoriated tungsten, the secondary emission coefficient is not a constant; it can change with the surface temperature. They estimate that for $\mathrm{Ar}^{+}$ions with energy ranging between 10 and $600 \mathrm{eV}$ and a surface temperature of $2000 \mathrm{~K}$ on a clean surface, the secondary emission coefficient could be set to 0.1 . Phelps and Petrović recommend that it should not exceed this value. Applying this data to the modeling of a tungsten cathode layer in the presence of argon shielding gas, Cayla [20] showed that the secondary electron emission dominates the thermo-field emission (RichardssonDushman-Schottky model) when the temperature of the plasma electrons in front of the cathode is up to about $7500 \mathrm{~K}$ for a plasma temperature of $10,000 \mathrm{~K}$. Cayla's calculations [20] indicate that this electron temperature corresponds to a cathode surface temperature up to about $2900 \mathrm{~K}$. Gonzales et al.'s [22] cathode layer calculations also show that for tungsten cathode, argon gas and current densities lower than $5 \times 10^{5} \mathrm{~A} \mathrm{~m}^{-2}$ (which is quite low compared with standard values of current density in GTAW), the value of the secondary emission coefficient has a significant influence on the cathode sheath drop voltage, the heat flux to the cathode, and the surface temperature of the cathode.

Electron emission at the cathode surface as well as ion recombination give rise to charge, ionization, and thermal non-equilibrium phenomena in the cathode layer. Each of these phenomena is associated with a specific characteristic length. The Debye length $\lambda_{D}$ is the scale over which the ions are screened by the electrons. The smallest mean free path $\lambda_{c}$ is the distance travelled by a charged particle before colliding with a similar or dissimilar charged particle [23]. The recombination length $\lambda_{r}$ is the scale at which the recombination and ionization rate is of the same order as the rate of variation of electron and ion density due to ambipolar diffusion. Finally, the electron energy relaxation length $\lambda_{e}$ represents the length over which electrons dissipate the energy they gain from the electric field while colliding with heavy particles (i.e., neutrals and ions). A gas commonly used in GTAW is argon. These characteristic lengths were evaluated by Benilov and Marotta for an argon plasma with a number density of about $10^{23} \mathrm{~m}^{-3}$ and temperatures of the order of $10,000 \mathrm{~K}$ [23]. These authors obtain a Debye length of the order of $10^{-8} \mathrm{~m}$, the smallest mean free path $\lambda_{c}$ of the order of $10^{-7} \mathrm{~m}$, a recombination length $\lambda_{r}$ of the order of $10^{-5} \mathrm{~m}$, and an electron energy relaxation length $\lambda_{e}$ of the order of $10^{-4} \mathrm{~m} \mathrm{[23].} \mathrm{This} \mathrm{last} \mathrm{length}$ is large due to the poor efficiency of energy exchange during collisions of particles with disparate masses. These orders of magnitude lead to

$\lambda_{D} \ll \lambda_{c} \ll \lambda_{r} \ll \lambda_{e}$

Based on this ordering, sub-regions of the cathode layer can be distinguished, each being defined by a specific scale and its related dominant physics. Notice that for an Ar plasma at much larger temperature or for a diatomic plasma (e.g., $\mathrm{N}_{2}$ plasma in [23]), this ordering may change to $\lambda_{D} \ll \lambda_{c} \ll \lambda_{e} \ll \lambda_{r}$. In the vicinity of the emitting region of a cathode surface, a molecular plasma is indeed characterized by a high dissociation degree.

According to the above ordering, the first sub-layer on the surface has a thickness of the order of the Debye length and is located in $0<x \leq \lambda_{D}$ as indicated in Fig. 2. It is assumed that the cathode surface is located in $x=0$ and the $x$-axis oriented toward the plasma column. As $x \leq \lambda_{D}$, local charge neutrality is not satisfied in this region, while it is satisfied further away from the surface. This first sub-layer is called the space charge layer, or the sheath, depending on the authors. For pressure (as 


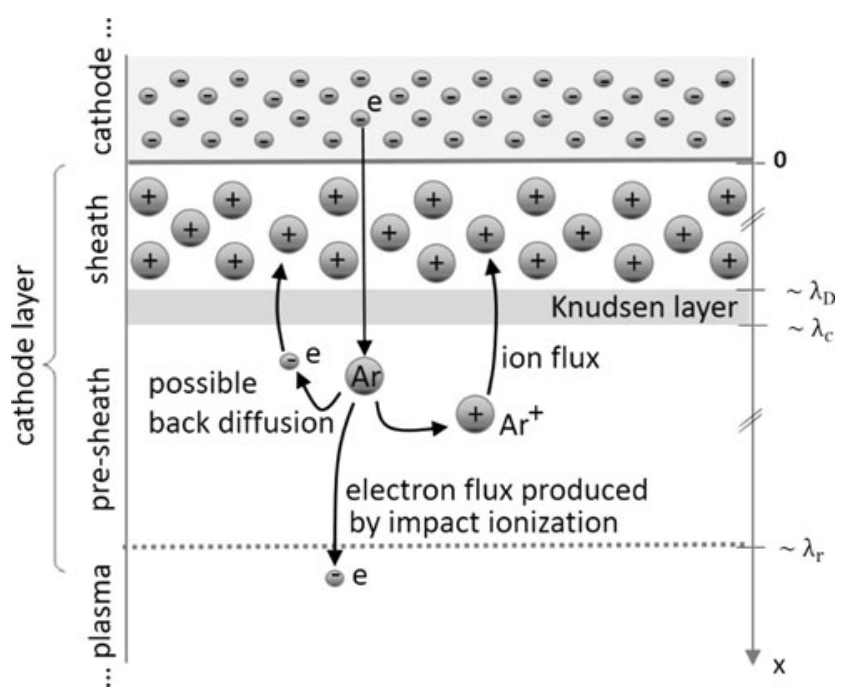

Fig. 2 Schematic diagram of sub-regions of a cathode layer in GTAW (the distances from the cathode surface are not at scale)

considered here) that is close to atmospheric pressure, the sheath thickness $\Delta x \approx \lambda_{D}$ is much smaller than the smallest mean free path $\left(\lambda_{\mathrm{c}}\right)$. The number of collisions in the sheath is then quite low (and in practice usually assumed to be negligible) so that local thermodynamic equilibrium cannot be defined. Therefore, the continuum approach is not valid and this sub-layer is modeled at the kinetic scale (usually collisionless kinetic). It implies also that diffusion cannot be the leading order phenomena in the very close vicinity of the cathode surface. The charged particles are then assumed to be freely accelerated by the electric field. This description was hypothetical and controversial within the frame of atmospheric and high pressure electric arcs until being rather recently confirmed by experimental observations (see Sect. 2.1). Notice that ionization and recombination are also negligible in the sheath, but ion recombination can occur at the cathode surface (at $x=0$ ).

The second sub-layer from the surface is the Knudsen layer (see Fig. 2). Its thickness is of the order of the smallest mean free path $\lambda_{c}$. The collision frequency in the Knudsen layer is thus too low to reach any partial local thermal equilibrium (LTE). It implies that this sub-layer is kinetic, contrary to the regions located further away from the surface for which a continuum approach (more specifically a hydrodynamic limit) is valid.

The third sub-layer from the cathode surface has a thickness of the order of the recombination length $\lambda_{r}$ Local ionization equilibrium is thus not satisfied in this sub-layer (ionization dominates over recombination, see Fig. 2), while it is satisfied further away from the cathode surface. This third sub-layer is called the ionization layer or the pre-sheath, depending on the authors. It is locally charge neutral (as $\lambda_{D} \ll \lambda_{r}$ ), and although the collision frequencies are large enough to justify a continuum approach, thermal equilibrium is only partial since $\lambda_{r} \ll \lambda_{e}$. In the thermal non-equilibrium region, the electron temperature $T_{e}$ is larger than the heavy particle temperature $T_{h}$. In general, the thermal non-equilibrium region does not coincide with the pre-sheath. For monoatomic species, such as argon at about $10,000 \mathrm{~K}$, the ordering $\lambda_{r} \ll \lambda_{e}$ indicates that LTE should be reached outside the pre-sheath (so within the plasma column) [23]. On the contrary for diatomic species (such as $\mathrm{N}_{2}$ discussed in [23]), LTE should be established within the pre-sheath since then $\lambda_{e} \ll \lambda_{r}$.

The role of these different sub-layers is now further detailed.

\subsection{Cathode sheath (or space charge layer)}

This thin region in contact with the cathode surface can be formed under the specific conditions given in Sect. 2.2. It makes the transition between metallic and gaseous conduction and is mainly made of positive ions attracted by the negative charges forming the surface potential barrier on the cathode surface. Its existence was difficult to confirm experimentally within the frame of atmospheric and high pressure arcs due to the extreme conditions that hinder direct observations. The confirmation was provided via indirect observation by Dabringhausen et al. [24], Luhmann et al. [25], Nandelstädt et al. [26], Lichtenberg et al. [27], as well as in more recent studies [28], [29]. The experimental data provided by these authors are also very useful for testing the models.

The sheath plays an important role in the cathode layer function [13]:

- It forms a potential barrier that enhances electron emission by lowering the cathode surface potential barrier.

- It accelerates the emitted electrons, pushing them further away from the cathode surface (toward the pre-sheath or ionization layer) and provides them with sufficient kinetic energy to promote impact ionization as they reach the presheath, and thus sustain the discharge.

- It accelerates the ions generated in the pre-sheath toward the cathode. These ions heat up the cathode to thermionic emission temperature by transferring their kinetic energy to the cathode while colliding with the cathode wall. Among these ions, the most highly energetic may also promote secondary emission.

- It forms a potential barrier that reduces the back diffusion of the electrons from the pre-sheath toward the cathode.

The charge fluxes taking place in the sheath are thus the flux of electrons emitted from the cathode surface by thermionic or thermo-field emission and by secondary emission, and also the flux of ions and back diffused electrons toward the cathode. The heat transfer to/from the cathode surface because of these charge fluxes thus include the following: 
- The heat lost by thermionic or thermo-field emission, by secondary emission, and

- The heat gain with the ions and back diffusion electrons crossing the sheath and reaching the cathode surface.

These charge and heat fluxes depend on the cathode surface temperature, the electron and ion temperature at the sheath/pre-sheath interface, the plasma composition at the sheath/pre-sheath interface, and the potential fall across the sheath $[4,20,30]$.

\subsection{Knudsen layer}

The Knudsen layer is the transition layer allowing a smooth matching between the charged and almost collision-free sheath and the quasi-neutral and partial LTE pre-sheath. Without this layer, the sheath/pre-sheath interface would present a singularity $[31,32]$. Ion losses due to recombination at the cathode surface indeed lead to a distortion of the ion distribution. As a result, the ion velocity at the sheath/pre-sheath interface needs to be larger than a critical threshold (called Bohm velocity) to be able to form the sheath [32]. The thermal velocity of the ions within the partial LTE pre-sheath cannot reach this critical value. The role of the Knudsen layer, which is kinetic and dominated by electric field acceleration rather than collisions (although collisional), is to accelerate the ions up to the Bohm velocity.

\subsection{Cathode pre-sheath (or ionization layer)}

The cathode pre-sheath is the region where charged species are produced by ionization, sustaining the electrical discharge. For pressures close to atmospheric pressure, it is usually assumed that impact ionization is the dominant ionization process [13]. The ionization energy is mainly provided by the impacting electron if the electron density is not too low. It can also result from the collision of a heavy particle (atom or ion) in an excited electronic state with an electron (as in the cold arc fringes). In that second case, both impinging particles contribute to providing the ionization energy. As sketched in Fig. 2, the ionization can occur when an electron, emitted from the cathode surface and accelerated through the sheath, collides with a gas atom or an ion in the pre-sheath. It results in two free electrons and a positively charged ion, providing a flux of ions and of back-diffusion electrons toward the sheath, as well as an electron flux toward the plasma. The steep gradients of particle densities present in this layer lead to particle diffusion. The thermal non-equilibrium leads to a decoupling of the electrical conductivity and the heavy particle temperature. Important physical characteristics of this layer are thus diffusion, thermal non-equilibrium (partial LTE), and ionization non-equilibrium (i.e., partial local chemical equilibrium or partial LCE), and these phenomena are concomitant.

\section{Cathode layer models for electric arc at atmospheric pressure}

The above listed cathode layer sub-regions are defined by specific scales and specific physics. The cathode layer problem can thus be formulated as a boundary layer problem including several one-dimensional sub-layers linked through a suited coupling procedure $[5,32]$. The boundary layer formulation is however based on the assumption that a sub-layer can be assumed one-dimensional if its thickness is small enough compared to the characteristic length of the geometry studied. This characteristic length could be the radius of the cathode tip. The boundary layer formulation is widely used for modeling the cathode sheath layer of high-intensity discharge lamps. The radius of cathode tips used in GTAW is usually smaller than for HID lamps and can be of the order of $\lambda_{r}$ (the thickness of the ionization layer).

Cathode layer models for electric arcs at atmospheric pressure can be organized in three groups, classified according to their approach for modeling the pre-sheath (or ionization layer): (1) the diffusion approach, (2) the partial LTE approach, and (3) the hydrodynamic approach. The models representative of these approaches are categorized in Table 1 with regard to the respective physical phenomena addressed. The main characteristic of these approaches are now summarized.

\subsection{The diffusion approach}

The diffusion approach was introduced by Lee et al. [33], without accounting for the arc-flow interaction, and by Morrow and Lowke [34], taking into account the arc coupling. It aims at providing a smooth transition of the electron current density from the cathode to the plasma column. The diffusion approach assumes that charge diffusion in the pre-sheath (or ionization layer) is the dominant cathode layer phenomena promoting arc-cathode coupling. With regard to the physics described in Sect. 2, the simplifications resulting from this assumption are as follows. The thermal non-equilibrium in the pre-sheath is assumed to be negligible compared to the non-equilibrium of charge densities. The pre-sheath is thus modeled assuming LTE (and thermal diffusion is neglected). The influence of the sheath (or space charge layer) and of the Knudsen layer is assumed to be negligible. The energy transported by the ion flux to heat the cathode surface and promote electron emission is then related to the thermal velocity in the pre-sheath rather than the Bohm velocity provided by the Knudsen layer. As the sheath is neglected, the electron emission is purely thermionic and it cannot be field enhanced. Also, the electrons and ions cannot be accelerated by the sheath fall voltage in order to convey energy from the electric field to the thermal plasma. Nevertheless, the diffusion approach models this energy transfer. 
Table 1 Categorization of cathode layer models (atmospheric thermal plasma)

$$
\text { Main properties of cathode layer model Appl. Some other properties }
$$

$\mathrm{S} \quad \mathrm{Kn} \quad \mathrm{PS} \quad \phi_{e m} \phi_{s e} \phi_{b d}$ Po Plasma $\chi$

\begin{tabular}{|c|c|c|c|c|c|c|c|c|c|c|c|}
\hline \multicolumn{12}{|l|}{ Diffusion approach } \\
\hline Morrow, Lowke (1993) [34] & - & - & $\mathrm{D}$ & $\mathrm{R}$ & - & - & - & LTE & - & GTA & \\
\hline Haidar (1999) [38] & - & - & $\mathrm{D}$ & $\mathrm{R}$ & - & - & & $2 \mathrm{~T}$ & - & GTA & \\
\hline Sansonnens et al. (2000) [37] & - & - & $\mathrm{D}$ & $\mathrm{R}$ & - & + & - & LTE & - & GTA & \\
\hline Lowke and Tanaka (2006) [43] & - & - & - & $\mathrm{R}$ & - & - & - & LTE & - & GTA & $\begin{array}{l}\text { large cells to neglect non-eq. } \\
\text { densities }\end{array}$ \\
\hline \multicolumn{12}{|l|}{ Partial-LTE approach } \\
\hline Zhou et al. (1994) [50] & + & - & $2 \mathrm{~T}$ & RS & - & + & + & LTE $^{1}$ & Richley, Tuma & & ${ }^{1}$ as boundary condition \\
\hline Coulombe (1997) [52] & + & $\mathrm{HB}$ & $2 \mathrm{~T}$ & RS & - & + & 1 & $\mathrm{LTE}^{2}$ & Richley, Tuma & & $\begin{array}{l}{ }^{1} \text { fixed cathodic fall voltage } \\
{ }^{2} \text { as boundary condition }\end{array}$ \\
\hline Cayla (2008) [20] & $\mathrm{B}$ & $\mathrm{HB}$ & $2 \mathrm{~T}$ & RS & + & + & + & LTE & van de Sanden & HID & \\
\hline Javidi et al. (2014) [55] & $\mathrm{B}$ & $\mathrm{HB}$ & $2 \mathrm{~T}$ & RS & + & + & + & LTE & van de Sanden & HID GTA & \\
\hline \multicolumn{12}{|l|}{ Hydrodynamic approach } \\
\hline Hsu, Pfender (1983) [63] & + & - & $\mathrm{H}^{1}$ & $\mathrm{RS}$ & - & + & + & $\mathrm{LTE}^{2}$ & Potapov & GTA* & $\begin{array}{l}{ }^{1} \text { expe. cathodic fall voltage as } \\
{ }^{2} \text { boundary condition }\end{array}$ \\
\hline Delalondre (1990) [11] & + & - & $\mathrm{H}^{1}$ & RS & - & + & + & $2 \mathrm{~T}$ & Potapov & GTA* & ${ }^{1}$ estimated cathodic fall voltage \\
\hline Benilov (1995) [23]-(2008) [4] & $\mathrm{B}$ & $\mathrm{HB}$ & $\mathrm{H}$ & RS & - & + & + & $2 \mathrm{~T}$ & $n_{e}$ and $T_{e}$ function & HID GTA* & \\
\hline Wendelstorf (2000) [71] & + & $\mathrm{HB}$ & $\mathrm{H}$ & RS & - & + & 1 & $2 \mathrm{~T}$ & $n_{e}$ and $T_{e}$ function & GTA & ${ }^{1}$ simplified to McKeown formula \\
\hline Schmitz, Riemann (2001) [31] & $\mathrm{B}$ & $\mathrm{CB}$ & $\mathrm{H}$ & RS & + & + & + & $\mathrm{LTE}^{1}$ & $T_{e}$ function ${ }^{2}$ & & $\begin{array}{l}{ }^{1} \text { as boundary condition } \\
{ }^{2} \text { van de Sanden type }\end{array}$ \\
\hline Baeva et al. (2012) [76] & $\mathrm{B}$ & HB & $\mathrm{H}^{1}$ & RS & - & + & + & $2 \mathrm{~T} x$ & $T_{e}$ function & HID GTA* & ${ }^{1}$ with plasma column \\
\hline
\end{tabular}

$S$ sheath modeled with voltage fall estimate (if + ) or with collisionless Boltzmann equation (if B), Kn Knudsen layer modeled with collisional Boltzmann equation (CB) or a Heaviside type function combined with Bohm criterion (HB), PS pre-sheath modeled with the diffusion (D) or partial LTE (2T) or hydrodynamic approach $(\mathrm{H}), \phi_{e m}$ thermionic electron emission with Richardson-Dushman law $(R)$ or field enhanced thermionic emission with Richardson-Dushman law and Schottky correction $(R S), \phi_{s e}$ secondary electron emission modeled (if + ), Po electric field at the cathode surface calculated solving Poisson equation (if +), Plasma plasma column model (same abbreviations as for PS), $\chi$ ionization model, Appl. application to HID lamp (HID), to GTA $(G T A)$, or similar $\left(G T A^{*}\right)$

This approach provides a non-equilibrium composition of the cathode layer based on ambipolar diffusion induced by the ion flux toward the cathode. It results in an electron density larger than the LTE density in the cathode vicinity, and thus promotes current flow via diffusion. Electrons and ions are thus accelerated by a potential fall induced by the ambipolar diffusion. However, as this ambipolar potential fall is low, the electrons cannot be accelerated up to a kinetic energy large enough to ionize the media and self-sustain the discharge (as described in Sect. 2). The discharge is instead sustained by the diffusionpromoted current flow and the resultant Joule heating.

To be applied, this approach requires knowledge of the electron density distribution. This distribution is calculated solving a diffusion equation accounting for charge production (ionization) and uses the cathode surface temperature as a boundary condition. This last one is known from the energy conservation equation, setting the total energy flux on the cathode surface as a boundary condition. The total energy flux includes the energy removed from the cathode surface while electrons are emitted and the energy gained by ion recombination at the cathode surface. Back diffusion electrons are neglected. The emitted electron flux is obtained from Richardson's law while the incoming ion flux is known from the ion current density.

The model proposed by Morrow and Lowke [34] was further developed over time (e.g., [12, 35-37]). In the original model [34], the ion current density is calculated implicitly. In the more recent development of Sansonnens et al. [37], the ion current density is changed to an explicit expression in order to satisfy conservation of the total particle flux density. The electrical conductivity is defined with an ambipolar diffusion term in [37] rather than with the cathode surface electric field in [34]. Sansonnens et al. underlined that for thoriated tungsten (and not for pure tungsten), distinct work functions should be used for modeling the thermionic electron flux and the thermionic electron energy flux from the cathode surface, in order to allow sufficient cooling of the cathode. They recommend using the work function of thoriated tungsten for the former and the work function of pure tungsten for the latter to obtain more accurate results [37]. 
Other modifications have been made, for example, to significantly reduce the computational time [36] and to introduce partial thermal equilibrium [38]. This model has been applied to several studies by e.g. Tanaka et al. [39], Ushio et al. [40], Lowke et al. [41], and Füssel et al. [42].

An alternative version of this approach called the "LTEdiffusion approximation" was developed by Lowke and Tanaka [43]. It allows rapid calculations avoiding solving the continuity equation for the electron density. As it implies, the physics of the pre-sheath is also neglected, as it does not aim at gaining insight into the cathode plasma coupling. This approximation is made using a mesh size in front of the cathode surface large enough to allow neglecting the region of non-equilibrium densities. Lowke and Tanaka emphasize that the choice of mesh size on the plasma side at the cathode surface is critical to reproduce realistic temperature fields in the arc. Today, the LTE-diffusion approximation seems to be the most popular for simulating the heat source in GTAW (see e.g., [2, 44-48]).

\subsection{The partial LTE approach}

The partial LTE approach is almost the opposite of the diffusion approach. It considers the thermal non-equilibrium (partial LTE) and large gradients in both temperature and electric field in front of the cathode, but it ignores diffusion. It also takes into account the sheath, the calculation of the plasma composition at the sheath/pre-sheath interface thanks to a two-temperature composition model, and it includes the electron back diffusion. Several variants were developed by Zhou et al. [50, 51], Coulombe and Meunier [52, 53] (notice that the latter was applied in a different context, namely non-refractory cathodes), and more recently, by Cayla [20, 22, 54] and Javidi $[30,55]$.

Zhou et al. neglect the Knudsen layer and assume that the energy transported by the ion flux to heat the cathode surface is related to the thermal velocity in the pre-sheath. In contrast, Coulombe and Meunier, Cayla, and Javidi take into account Bohm's criterion (as proposed by Benilov, see next section) and thus the ion acceleration provided by the Knudsen layer.

For Coulombe and Meunier, the cathode sheath potential fall $U_{s}$ is an input parameter set based on experimental evaluations. Conversely, for Zhou et al., Cayla, and Javidi, the potential fall $U_{s}$ is an output of their model. The sheath model aims at calculating locally $U_{s}$ and the cathode surface temperature. This model is made of two equations, namely the energy conservation and the current density conservation at the cathode surface. To be solved, this system needs to be supplemented with data for expressing the boundary conditions. These data are the total local current density and the plasma composition as well as the electron and heavy particle temperatures at the sheath/pre-sheath interface. Most of the authors assume that the ion (or heavy particle) temperature at the sheath/pre- sheath interface is equal to the cathode surface temperature since the sheath is almost collisionless.

The plasma composition as well as the electron temperature at the sheath/pre-sheath interface is provided by the pre-sheath model. At this level, Zhou et al. proceed differently from Cayla and Javidi. Zhou et al. calculate the plasma composition based on the approach of Richley and Tuma [56]. They determine the electron temperature applying Steenbeck's minimization principle [57]. Notice that it has been shown since then that Steenbeck's minimization principle should not be considered as a corollary of the principle of minimum entropy production [58].

Cayla [20] showed that the input parameters used by Benilov [4] for the cathode layer model, namely the cathode fall voltage and cathode surface temperature, could be replaced by the current density. The current density is indeed more convenient for coupling the cathode, cathode layer, and plasma column. The pre-sheath model of Cayla and Javidi is based on conservation principles: energy conservation, charge conservation, nucleus conservation, and detailed balance for each type of ionization/recombination involved. To close this system, relations linking the direct and reverse reaction rates are needed for each ionization/recombination reaction involved. They use the van de Sanden formulation [59] of the Saha law since it is consistent with their LTE assumption for the plasma column, and it satisfies the second law of thermodynamics [60]. The resultant system of equations describing their pre-sheath model includes energy conservation, electric neutrality, Dalton's law, and a set of three Saha laws for determining the Ar atoms and $\mathrm{Ar}+, \mathrm{Ar} 2+$, and $\mathrm{Ar} 3+$ ion densities $[20,30]$. The systems of equations modeling the sheath and the pre-sheath are non-linear and can be solved numerically using an iterative procedure.

The cathode layer models differ also in other modeling aspects. For instance, Cayla and Javidi take into account secondary emission while most of the other authors do not (see column ' $\phi_{\text {se }}$ ' in Table 1).

The partial LTE approach was first applied to study the cathode sheath alone (assuming conditions in the cathode and plasma column). To our knowledge, the cathode layer models of Zhou et al. and Coulombe and Meunier have not been applied to the coupling of cathode and arc for studying GTAW. However, several applications of the model by Zhou et al. to other domains such as HID lamps can be found. The more recent cathode layer model by Cayla and Javidi are coupled with the cathode and plasma column. They have been applied to HID lamps in [20, 22, 55].

\subsection{The hydrodynamic approach}

The hydrodynamic approach is the most complete with respect to the cathode layer physics. It is based on a hydrodynamic description of the pre-sheath and thus includes both 
thermal non-equilibria and species diffusion. The partial LTE approach can be seen as a simplified version of the hydrodynamic approach. In contrast, the diffusion approach is quite different in essence. The diffusion limit is a standard technique in continuum fluid mechanics for studying boundary layer problems. Diffusion (also called drift-diffusion) and hydrodynamic limits can be derived from the same set of Boltzmann kinetic equations but with a different scaling in time $t$, while maintaining the same scaling in space $x$. Using an appropriate scaling of the small parameter $\varepsilon$ (e.g., in plasma physics, $\varepsilon^{2}$ is the ratio of electron to heavy particle mass), the diffusion approximation and the hydrodynamic limit can respectively be derived by letting the small parameter $\varepsilon$ tend to zero [61, 62]. The diffusion scaling is $\varepsilon: t \rightarrow \varepsilon^{2} t, x \rightarrow \varepsilon x$, while the hydrodynamic scaling is $\varepsilon: t \rightarrow \varepsilon t, x \rightarrow \varepsilon x$. In such a scaling, $t$ represents the typical time for an information to be transported over a distance $x$. A consequence of the diffusion scaling is to move the collisional operator for impact ionization at a higher order (in terms of $\varepsilon$ ) in the rescaled kinetic model, and thus make it vanish when going to the macroscopic limit [61]. This operator is the source of the ionization nonequilibrium characteristic of the pre-sheath. These approaches are valid if a sufficiently large number of collisions between electrons and collisions between heavy particles take place to allow passing to the macroscopic limit for both light and heavy species. This condition is verified in the ionization layer since the characteristic mean free paths of neutrals, ions, and electrons are lower than $\lambda_{r}$ but not in the closer vicinity of the wall (i.e. in the sheath).

Several variants of the hydrodynamic approach were introduced and further improved by Hsu and Pfender [63], Delalondre [11, 64], Benilov [4, 6, 7, 23, 25, 65-70], Wendelstorf [71-73], Schmitz and Riemann [31, 74], and Baeva $[3,70,75-78]$ for modeling the pre-sheath. The extensive contribution of Benilov to the development of a selfconsistent cathode layer model was partly used by several other authors including Wendelstorf and Baeva (also Cayla and Javidi). All these variants consider field-enhanced thermionic emission and thus the cathode sheath. All consider at least three types of current densities: thermionic emission, back-diffusion electrons, and positive ions. However, they differ in many other aspects.

In the models by Hsu and Pfender, and by Delalondre, the sheath is considered but not modeled and the Knudsen layer is not considered. The ion velocity at the sheath/pre-sheath interface is assumed to be the thermal velocity. In other words, Bohm's criterion is not satisfied. The cathode sheath potential fall $U_{s}$ is set as an input parameter. In contrast, the model proposed by Schmitz and Riemann is the most detailed for describing the Knudsen layer. This is indeed the only one modeling this region with a collisional Boltzmann equation. The Knudsen layer model developed by Benilov is an analytic model with an ion distribution function at the sheath/pre- sheath interface given by a Heaviside function bounded to verify Bohm's criterion. An advantage is its much lower computational cost compared to a full kinetic approach. Nandelstädt et al. [26] showed that the results of the detailed Knudsen layer model of Schmitz and Riemann are close to the results obtained with Benilov's simplified model. The sheath models of Benilov, Wendelstorf, and Baeva are very similar to the description given in Sect. 3.2 for Cayla's and Javidi's sheath model. However, slightly different coefficients can be found from author to author in the formulations of the conservation equations. These differences are not detailed here.

Important differences between the models belonging to the hydrodynamic approach concern the modeling of the presheath. The energy conservation equations for electrons and heavy species can differ, as well as the species taken into account in the Ar-plasma which is investigated by all the authors. Benilov and Baeva seem to neglect the ions $\mathrm{Ar}^{2+}$ when calculating the Ar-plasma composition and the thermodynamic and transport properties. On the contrary, Delalondre and Wendelstorf take into account $\mathrm{Ar}^{2+}$. Delalondre showed that $\mathrm{Ar}^{2+}$ has a very significant influence on properties such as the specific heat for plasma temperatures above $11,000 \mathrm{~K}$ in [11]. In a more general way, the thermodynamic and transport coefficients are in several cases based on different methods. Rat et al. [79] showed that some methods can lead to significant discrepancies in the results. Besides, some of the studies are based on Devoto's diffusion model. It is now known that this model does not satisfy mass conservation [79].

Another example of important difference lies in the reaction processes and the closure relations linking the direct and reverse reaction rates. Schmitz and Riemann model impact ionization (and its reverse recombination) assuming that all the ionization energy is provided by the impacting electron. Their closure relation is similar to van de Sanden formulation of the Saha law. This type of ionization is considered to be dominant in hot regions where the electron density is large enough. In colder regions, a significant part of the ionization energy may need to be provided by the particle to be ionized. Conversely, Hsu and Pfender, Delalondre, and Wendelstorf consider only the ionization reactions with incident heavy particles in an excited electronic state. The closure relations used by Hsu and Pfender and Delalondre are given by Potapov generalization of the Saha law [80]. Van de Sanden [59] showed that Potapov formulation is not thermodynamically correct. This can also be motivated based on kinetic theory [60]: Potapov formulation does not satisfy the entropy inequality (second law of thermodynamics). A consequence for models violating the entropy inequality is that they can lead to unphysical solutions. A clear illustration can be seen in the context of rarefied gas dynamics in [81]. Benilov considers reactions covering both electron impact ionization/ recombination (similar to Schmitz and Riemann) and the decay of excited states due to radiation emission. As a result, the 
reaction rates depend on temperature and on the electron density. It implies that the resultant closure relation is not a Saha law. Baeva uses the most complete set of reactions. It includes the two previous types of impact ionization, also impact ionization between two heavy particles, and radiative recombination [75].

The models describing the plasma column can also differ. In the models by Delalondre, Wendelstorf, and Benilov (the one coupled with the arc), the plasma is in partial LTE and at chemical equilibrium. In the model developed by Baeva, the plasma core is in partial LTE and also in partial LCE. It implies that for the former models, the pre-sheath is part of the cathode layer, while for Baeva, the pre-sheath is modeled with the plasma column rather than as a boundary sub-layer. In other words, the pre-sheath is not simplified to a one-dimensional approximation in that latter case.

Due to the above mentioned differences, the plasma model of Baeva taken in the limit of chemical equilibrium may not relax toward the plasma column model of Benilov for instance (when considered independently of the cathode layer model). Similarly, the plasma column model of Benilov taken in the limit of thermal equilibrium may not relax toward the plasma model of Cayla (again when considered independently of the cathode layer model).

The models proposed by Hsu and Pfender, Schmitz and Riemann, as well as the first developments by Benilov, were applied to the study of the cathode layer alone. The more recent studies by Benilov, as well as the models of Delalondre, Wendelstorf, and Baeva, couple cathode, cathode layer, and plasma column and were applied to HID lamps and GTAW, as indicated in the column 'Appl.' (Applications) in Table 1.

\section{Results provided by cathode layer models coupled with cathode and plasma column}

The previous section and Table 1 illustrate the diversity of models used for simulating GTAW when accounting for the cathode layer coupling. Most of these models have been tested by comparison with experimental results. Very recently, model comparisons have also been conducted. Finally, parts of these models have been applied to parametric studies in order to gain a deeper understanding of the cathode layer coupling process. Some of these results are now summarized.

\subsection{Comparative studies}

Most of the models representative of the diffusion, the partial LTE, and the hydrodynamic approach have been tested by comparison with experimental data, as indicated in Table 2. It is clear from this table that the models belonging to distinct approaches are seldom tested with the same experimental

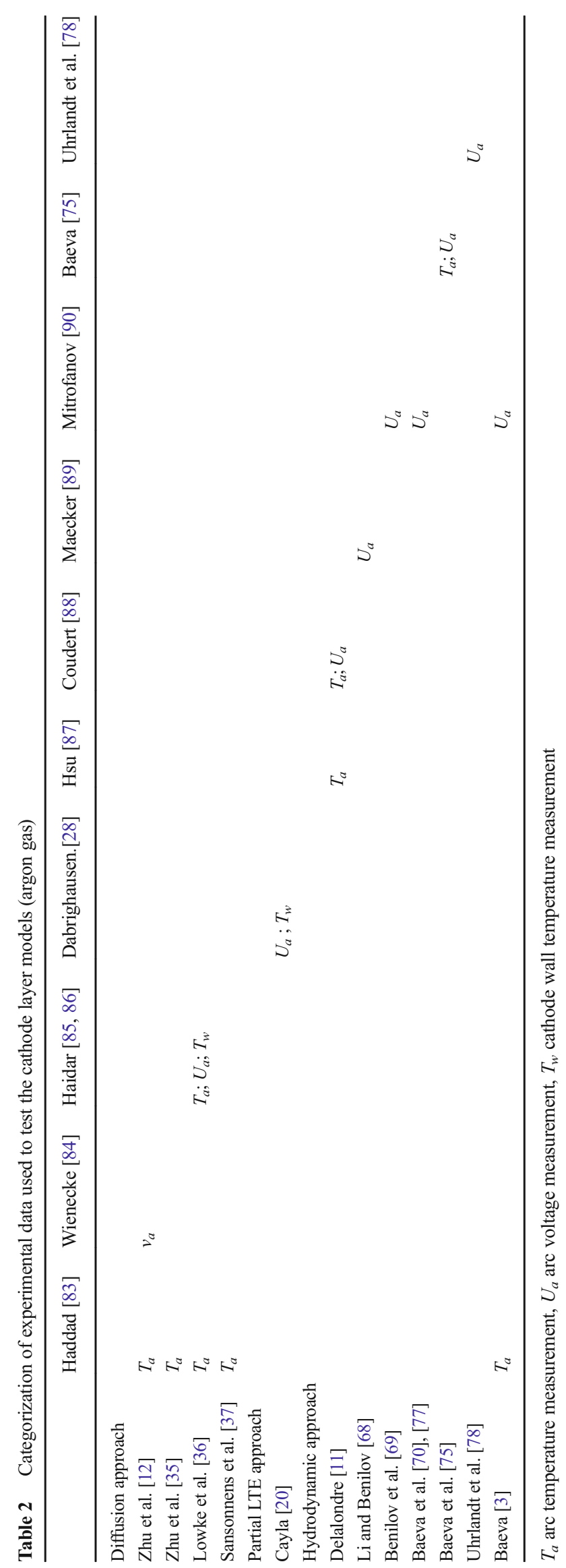


data. This would have been very convenient for comparing the cathode layer models, although other differences may complicate the task. For instance, the models based on the diffusion approach often neglect the radial component of the current density when calculating the magnetic field, while the models based on the partial-LTE and the hydrodynamic approach do not. This additional difference could also have an influence on the fields in the arc, as underlined in [82].

The cathode layer models based on the diffusion approach of Morrow and Lowke [34] are applied (in [12, 35-37]) to a GTAW configuration studied experimentally by Haddad and Farmer [83]. The related process parameters are given in Table 3. In the first application (see [12]), discrepancies between calculated and measured arc temperature are observed, mainly in the anode vicinity. In the more recent applications made with an improved model, very good agreement is observed [37].

The maximum plasma velocity calculated in [12] (about $500 \mathrm{~m} / \mathrm{s}$ ) is also compared with the experimental measurements of Wienecke [84]. The calculation results overestimate the experimental measurements by about $200 \mathrm{~m} / \mathrm{s}$. However, this comparison should be taken with caution since the process parameters were different. For instance the cathode used for the experiment was made of another material (graphite).

The same configuration is studied in [36] varying the current from 50 to $400 \mathrm{~A}$, the cathode tip angle $\left(16^{\circ}\right.$ and $\left.60^{\circ}\right)$, and the cathode material (pure tungsten and thoriated tungsten). The experimental measurements of Haidar and Farmer [84, 85] are used in [37] for testing calculation results: the maximum arc temperature, the overall arc voltage, and the cathode tip temperature. For both pure and thoriated tungsten electrodes, calculated and measured arc temperatures are in good agreement for the $60^{\circ}$ tip angle and the high current range. Discrepancies are observed with the $16^{\circ}$ tip angle, also at high current (200 A). The calculated arc voltage underestimates the measured voltage by about $2 \mathrm{~V}$; the authors assume that this difference could correspond to the cathode fall voltage neglected by their model.

The self-consistent cathode layer model coupled with an LTE plasma column (LTE-CL model) by Cayla is applied in [20] to an HID lamp studied experimentally by Dabringhausen et al. [28]. The cathode has a flat tip, and the argon pressure $(0.26 \mathrm{MPa})$ is not atmospheric, contrary to all the other test cases. The calculated cathode fall voltage and energy absorbed by the cathode are in good agreement with the experimental measurements. The calculated surface temperature overestimates the experimentally measured temperature by about $250 \mathrm{~K}$; this result is considered to be within the limits of experimental error [20].

The cathode layer model based on the hydrodynamic approach of Delalondre is applied to a GTAW configuration in [11]. The calculations are done prescribing the cathode sheath voltage and the size of the arc attachment based on experimental observations since this model is not yet self-consistent, contrary to the more recent models developed by Benilov, Cayla, and Baeva. The plasma temperature fields obtained at 200 and $300 \mathrm{~A}$ are in good agreement with the experimental measurements of Hsu et al. [87]. Comparisons are also made for the three following sets of electrical current and anode to cathode distance: $275 \mathrm{~A}, 27.3 \mathrm{~mm} ; 280 \mathrm{~A}, 50.6 \mathrm{~mm}$; and $287 \mathrm{~A}, 23.1 \mathrm{~mm}$ with the arc temperature and total arc voltage measured by Coudert and Grimaud [88]. A good qualitative agreement is observed.

The self-consistent cathode layer model developed by Benilov coupled with a partial LTE plasma column model (2T-CL model) is tested with the experimental measurements of Maecker [89] in [68]. The test case corresponds to a cylindrical column of a wall-stabilized dc arc in argon at atmospheric

Table 3 Categorization of experimental data used to test the cathode layer models

\begin{tabular}{|c|c|c|c|c|c|c|c|}
\hline & Appl. & Mat. & $\mathrm{d}(\mathrm{mm})$ & $\Theta$ & $\mathrm{L}(\mathrm{mm})$ & I (A) & Other \\
\hline \multicolumn{8}{|l|}{ Diffusion approach } \\
\hline Zhu et al. [12, 35, 37] & $G T A$ & $T h W$ & 3.2 & $60^{\circ}$ & 5 & 200 & \\
\hline Lowke et al. [36] & GTA & $T h W$ & 3.2 & $16^{\circ}, 60^{\circ}$ & 5 & $50 \ldots 400$ & \\
\hline \multicolumn{8}{|l|}{ Partial LTE approach } \\
\hline Cayla [20] & $H I D$ & $W$ & 1 & $180^{\circ}$ & 20 & $1.5 \ldots 5$ & $0.26 \mathrm{MPa}$ \\
\hline \multicolumn{8}{|l|}{ Partial LTE approach } \\
\hline Hsu and Pfender [63] & & $W$ & & $180^{\circ}$ & & 200 & No plasma coupling \\
\hline Delalondre [11] & $G T A^{*}$ & $W$ & & $60^{\circ}$ & 10 & $275 \ldots 287$ & \\
\hline Delalondre [11] & $G T A^{*}$ & $W$ & 10 & $40^{\circ}, 60^{\circ}$ & $23 \ldots 50$ & $275 \ldots 287$ & \\
\hline Li and Benilov [68] & $H I D$ & $W$ & 2 & $180^{\circ}$ & 10 & $10 \ldots 60$ & \\
\hline Benilov et al. [69] & $G T A^{*}$ & $W$ & 2 & & 10 & $20 \ldots 200$ & Hemispherical \\
\hline Baeva et al. [70], [77] & $G T A^{*}$ & $W$ & 2 & & 10 & $20 \ldots 200$ & Hemispherical \\
\hline Baeva et al. [75] & $G T A^{*}$ & $L a W$ & 2 & & 8 & 200 & Hemispherical \\
\hline
\end{tabular}

Appl. application, Mat. cathode material, $d$ cathode diameter, $\Theta$ cathode tip angle, $L$ distance between electrodes, $I$ current 
pressure. The deviation between calculated and experimental results is less than $6 \%$ for a current varying from 10 to $60 \mathrm{~A}$ [68]. A comparison using the more recent arc voltage measurements of Mitrofanov and Shkol'nik [90] covering the higher currents used in GTAW is made in [69]. The calculated arc voltage $U_{\text {arc }}$ and the measured voltage are observed to vary similarly with the current $(20$ to $200 \mathrm{~A}) . U_{\text {arc }}$ is defined here as the sum of the calculated plasma column voltage and the calculated cathode fall voltage $U_{s}$ along the symmetry axis. Very good agreement is again obtained at low current. At higher current, $U_{\text {arc }}$ overestimates the experimental arc voltage up to about $2 \mathrm{~V}$ (at $175 \mathrm{~A}$ ). The calculated cathode fall voltage is a minimum at high current (about $10 \mathrm{~V}$ at $200 \mathrm{~A}$ ). The minimum $U_{s}$ is however much larger than the cathode layer voltage of about $2 \mathrm{~V}$ evaluated with the diffusion approach.

These configurations are also used in $[68,69]$ to compare the self-consistent 2T-CL model with a simplified model: LTE arc without cathode layer. This simplified model requires boundary conditions on the cathode surface set using the calculation results of the self-consistent 2T-CL model. The temperature (also in the LTE plasma core) and arc voltage calculated with the two models are significantly different. The calculations show that the LTE model overestimates the resistance in the plasma bulk and in the cathode vicinity. This overestimation seems to somehow compensate the lack of cathode fall voltage in the LTE model.

Calculation results obtained with the more recent model of Baeva (cathode sheath model with thermal and chemical nonequilibrium plasma column, or 2TC-CL model) are also compared in [75] with experimental measurements and with an LTE model (with no cathode layer, and a uniform cathode surface temperature imposed at $3500 \mathrm{~K}$ ). The comparison confirms the earlier observation made by $\mathrm{Li}$ and Benilov [68] and Benilov et al. [69]. The cathode voltage drop obtained with the 2TC-CL model is about $9.5 \mathrm{~V}$. This is the value at the cathode tip (as in $[68,69]$ ) and not an effective voltage as in Cayla [20]). The arc voltage (13 V) calculated with the LTE model significantly overestimates the one calculated with the 2TC-CL model $(8.5 \mathrm{~V})$. The experimental voltage is closer to the non-equilibrium result $(18 \mathrm{~V})$ than to the LTE arc voltage. Conversely, spectroscopic measurements show that the 2TCCL model underestimates the measured temperature while the LTE model leads to a better agreement.

The 2TC-CL model is compared with the 2T-CL model developed by Benilov (its original implementation with thermal non-equilibrium and chemical equilibrium plasma column) in [70] and with the LTE-CL model developed by Cayla (a new implementation of it) in [77].

For a current lower than $100 \mathrm{~A}$ the 2T-CL model is closer to the experimental results of Mitrofanov and Shkol'nik [90] than the 2TC-CL model. In contrast, for currents from $100 \mathrm{~A}$ and above, the results of the 2TC-CL model are close to the experimental results while the 2T-CL and the LTE-CL model overestimate these results (up to $14 \%$ when the discrepancy is the largest). The LTE-CL model produces (as expected due to the LTE assumption in the plasma fringes) a more constricted arc than the 2TC-CL model. A similar trend is observed with the 2T-CL model. These discrepancies could have several causes since the models compared differ in several aspects that can either be related to the modeling (see Sect. 3.3) or to constraints imposed by the computational software used (see [70]). A cathode layer model with the sheath and the pre-sheath described as a boundary sub-layer (as described by Benilov, Cayla, and Javidi) or simply the sheath (as described by Baeva) is indeed a complex boundary condition and its implementation requires having sufficient access to the source code of the computational software.

These comparisons seem to indicate that when applied to GTAW, the diffusion approach provides good predictions of the arc temperature while the arc voltage is underestimated, and on the contrary, the hydrodynamic approach provides good predictions of the arc voltage while the arc temperature is underestimated.

\subsection{Parametric studies and cathode layer process understanding}

Several of the models listed in Table 1 have also been applied to do parametric studies in order to gain a deeper understanding of the cathode layer coupling process. Some of these results are now recalled.

The effect of back diffusion of electrons to the cathode for a 200-A arc is studied in [36] by Lowke et al. They observe that the total arc voltage is increased by $1.5 \mathrm{~V}$ (and infer that the sheath voltage is then increased from 1.5 to $3 \mathrm{~V}$ ) when accounting for electron back diffusion.

The influence of thermal non-equilibrium and diffusion in the pre-sheath is investigated in [63] by Hsu and Pfender. They apply their cathode layer model (hydrodynamic approach) to a 200-A free-burning argon arc and a thoriated tungsten cathode. The plasma column is included through boundary conditions set based on former studies (plasma temperature of 21,000 K for instance). As the authors use the diffusion approach, they observe a large deviation of the electron density calculated in the cathode vicinity compared to an LTE density. In [63], this deviation is mainly explained by ionization (rather than diffusion). It is combined with a very high generation of ions. The calculation results show large thermal non-equilibria across the cathode layer, with an electron temperature larger than the ion temperature. These deviations from LTE are associated with a cathode sheath voltage drop of about $4.5 \mathrm{~V}$ (thus larger than the ambipolar sheath voltage provided by the diffusion approach). The region presenting thermal equilibria (about $9 \times 10^{-5} \mathrm{~m}$ thick) is also slightly more extended than the region in ionization non-equilibrium (about $7.5 \times 10^{-5} \mathrm{~m}$ thick). In the cathode vicinity, it is observed that the fluxes of charged particles are mainly driven by the sheath 
voltage drop in the cathode vicinity. Thermal diffusion becomes important further away. For electrons, the potential gradient on the one hand and the density and temperature gradients on the other hand push the electrons toward opposite directions (respectively away and toward the cathode). Concerning the ions, all these gradients push them toward the cathode. Hsu and Pfender also observed that the ions generated in the pre-sheath and accelerated toward the cathode by the sheath voltage lead to a relatively high ion current (about $18 \%$ of the total current) close to the cathode.

Delalondre [11] applied her coupled cathode plasma model activating different parts step by step in order to investigate the significance of some physical phenomena. In one of these numerical tests, the sheath is omitted and replaced by a simple boundary condition on the electron density. It is observed that this simplification does not allow reproduction of the strong variation in electron density and in electric field in the vicinity of the cathode wall which is characteristic of the cathode layer.

The influence of the ionization non-equilibrium in the presheath is also studied in [11]. It is observed that a pre-sheath modeled assuming ionization equilibrium leads to a presheath electron temperature significantly larger than the heavy particle temperature but almost uniform, and it does not allow reproduction of the electron density gradient characteristic of the pre-sheath.

The influence of the electron enthalpy transport is also investigated in [11]. It is observed that although this transport has little influence on most of the arc temperature (it contributes to a rise of about $5 \%$ ), it plays a non-negligible role in the vicinity of the cathode tip as well as along the arc axis (regions characterized by high current density and large temperature gradient).

Cayla notes in [20] (page 75) that models for three-body recombination coefficient (useful for determining the plasma composition in the pre-sheath) can vary by up to four orders of magnitude depending on the underlying theory, e.g., Benilov [91] and Hinnov and Hirschberg [92]. It is observed that this coefficient has a significant influence on the current density and the cathode fall voltage due to its influence on the ion density at the sheath/pre-sheath interface. The current density calculated with the three-body recombination coefficient of [91] is an order of magnitude lower than with the coefficient of [92], which is in turn an order of magnitude lower than with van de Sanden formulation of the Saha law.

The influence of the thickness used in the LTE-CL calculations for describing the pre-sheath is studied in [20] (chapter 5) for a tungsten cathode and an argon gas (HID configuration). Two different conditions are studied: a uniform presheath thickness of $200 \mu \mathrm{m}$ (as often retained for HID lamp simulations) and $500 \mu \mathrm{m}$. It is observed that the thickness of the pre-sheath has a significant influence on the electron temperature at the sheath/pre-sheath interface, thus on the charge density in the pre-sheath, on the plasma temperature, on the electrical conductivity (in this case, a two-temperature model), on the extent of the conducting region at the cathode surface and finally on the fields within the arc (not only in the cathode vicinity). In practice this thickness may also be expected to vary locally along the cathode surface if the cathode surface temperature is not uniform.

The influence of the emitted current density on the cathode fall voltage is also investigated in [20]. The cathode fall voltage increases when the ratio of emitted to total current density decreases, in order to accelerate sufficiently the emitted electrons to promote ionization in the pre-sheath and compensate the decrease in emitted electrons with an increased number of charge carriers. The cathode fall voltage is observed to be significantly influenced by the thermal boundary condition on the top of the cathode and by the secondary emission coefficient.

A study of the influence of the extent of the arc attachment is performed in [70] by Beava et al. considering two cases: free and restricted attachment. It is observed, as earlier by Cayla [20] that the size of the arc attachment has a significant influence on the plasma temperature field. It can reach up to $4000 \mathrm{~K}$ difference $1 \mathrm{~mm}$ away from the cathode tip in the test cases investigated in [70]. It is concluded that a restricted attachment provides a better agreement with experimental measurements.

\section{Conclusions}

A large diversity of models coupling cathode, cathode layer, and plasma column has been developed during the past 35 years. They can be organized in three groups based on the approach used for modeling the pre-sheath or ionization layer: (i) the diffusion approach, (ii) the partial LTE approach, and (iii) the hydrodynamic approach. The ordering from (i) to (iii) is associated with an increasing consideration of the physics taking place in the cathode layer. Hopefully, most of these models should be represented in this review.

Several of these models have been applied to the study of GTAW. The variety of models and the modeling results obtained up to now show that this field is still under development. The review of the existing results indicates that the diffusion approach seems to be more accurate for evaluating the temperature fields in the plasma column while the hydrodynamic approach is more accurate for predicting the arc voltage. Plasma temperature and arc voltage belong to the very few quantities accessible from experimental measurements. It is known for instance that the heat transferred to the base metal, which is of great interest for welding applications, is not restricted to the heat flux due to temperature gradients. The heat flux due to current density can also be significant. However, the current density distribution in the arc is seldom discussed when applying a cathode layer model coupled with 
the plasma column, most probably due to the lack of experimental data. Measurements of the velocity field in the arc and pressure force on the base metal [42] would be useful, as they would provide (although indirectly, via the magnetic pinch force) information on the current density distribution.

The diffusion approach seems thus to be more accurate for evaluating the temperature fields in the plasma core. However, its applications have been on a rather restricted number of cathode geometries. It was observed in [36] that a very small cathode tip angle does not lead to results as satisfactory as the standard $60^{\circ}$ cathode tip angle. A more extended investigation of the influence of the process parameters on the ability of models to reproduce experimental results would be interesting too, without restriction on the model used.

Although the hydrodynamic approach is the most complete for describing the physics of the cathode layer, it seems to underestimate the temperature in the plasma column. According to [70], it is necessary to restrict the arc attachment area in order to reproduce a plasma temperature in good agreement with the experimental observations. The comparisons between models belonging to the partial LTE approach and the hydrodynamic approach recently developed by Baeva and co-workers might help to understand the cause of the extended arc attachment. However, the existing models differ in too many aspects (see Sect. 3.3) to draw clear conclusions. It would be interesting to know whether these different models (such as the plasma model with partial LTE and chemical nonLCE and the model with partial LTE and equilibrium ionization) can relax toward the same equilibrium (e.g., at ionization equilibrium). Physical phenomena such as metal vaporization at the cathode surface are usually neglected. It is known that a very small amount of metal ions in a plasma can significantly change the electrical conductivity. However, it is not yet known whether the small amount of metal vapor present in the cathode vicinity could be sufficient to influence the arc attachment.

Finally, the main issue may be the need for experimental data allowing further validation of the models. However, experimental observation is a challenge due to the high temperatures combined with the very small size of the cathode layer.

Acknowledgments This work was supported by KK-foundation in collaboration with ESAB. This support is gratefully acknowledged.

\section{References}

1. Murphy AB (2015) A perspective on arc welding research: the importance of the arc, unresolved questions and future directions. Plasma Chem Plasma Process 35:471-489

2. Gleizes A, Gonzales JJ, Freton P (2005) Thermal plasma modelling. J Phys D Appl Phys 38:R153-R183
3. Baeva M (2015) Thermal and chemical non-equilibrium effects in free-burning arcs, plasma chemistry and plasma processing, in press (online since 09/09/2015).

4. Benilov MS (2008) Understanding and modelling plasma-electrode interaction in high-pressure arc discharges: a review. J Phys D Appl Phys 41(14):144001 (30pp)

5. Franklin RN (2003) The plasma-sheath boundary region. J Phys D Appl Phys 36(22):R309-R320

6. Almeida NA, Benilov MS (2012) Physics of the intermediate layer between a plasma and a collisionless sheath and mathematical meaning of the Bohm criterion. Phys Plasmas 19:073514 (10pp)

7. Almeida NA, Benilov MS, Nadis GV (2008) Unified modelling of near-cathode plasma layers in high-pressure arc discharges. J Phys D Appl Phys 41:245201 (26pp)

8. Heberlein J, Mentel J, Pfender E (2010) The anode region of electric arcs: a survey. J Phys D Appl Phys 43(2):023001 (31pp)

9. Shkol'nik SM (2011) Anode phenomena in arc discharges: a review. Plasma Sources Sci Technol 20:013001 (31pp)

10. Ecker G (1961) Electrode components of the arc discharge. Ergebnisse Exakten Naturwissenschaften 33:1-104

11. Delalondre C (1990) Modélisation aérodynamique d'arcs électriques à forte intensité avec prise en compte de déséquilibre thermodynamique local et du transfert thermique à la cathode, $\mathrm{Ph}$.D. thesis. Rouen University, France

12. Zhu P, Lowke JJ, Morrow R (1992) A unified theory of free burning arcs, cathode sheaths and cathodes. J Phys D Appl Phys 25:12211230

13. Vacquié S (2000) L'arc électrique. Sciences et techniques de l'ingénieur, CNRS Editions.

14. Coulombe S, Meunier JL (1997) Themofield emission: a comparative study. J Phys D Appl Phys 30:776-780

15. He ZH (1995) Contribution à l'étude théorique et expérimentale de l'interaction plasma-cathode dans un arc électrique, Ph.D. Thesis. Pierre and Marie Curie University (Paris VI), France

16. Tanaka M, Ushio M, Ikeuchi M, Kagebayashi Y (2005) In situ measurements of electrode work function in free-burning arcs during operation at atmospheric pressure. J Phys D Appl Phys 38:29 35

17. Aillero Marín JA (2009) Estudio del comportamiento de cátodos termoiónicos en arcos de plasma. Ph.D. Thesis. Córdoba University, Spain

18. Sillero JA, Ortega D, Muñoz-Serrano E, Casado E (2010) An experimental study of thoriated tungsten cathodes operating at different current intensities in an atmospheric-pressure plasma torch. $\mathrm{J}$ Phys D Appl Phys 43:185204 (8pp)

19. Bergner A, Westermeier M, Ruhrmann C, Awakowicz P, Mentel J (2011) Temperature measurements at thoriated tungsten electrodes in a model lamp and their interpretation by numerical simulation. $\mathrm{J}$ Phys D Appl Phys 44:505203 (15pp)

20. Cayla F (2008) Modélisation de l'interaction entre un arc électrique et une cathode. PhD. Thesis. Laboratory Laplace, Paul Sabatier Univiversity, Toulouse

21. Phelps AV, Petrović ZL (2009) Cold-cathode discharges and breakdown in argon: surface and gas phase production of secondary electrons. Plasma Sources Sci Technol 8:R21-44

22. Gonzalez J-J, Cayla F, Freton P, Teulet P (2009) Two-dimensional self-consistent modelling of the arc/cathode interaction. J Phys D Appl Phys 42:145204 (14pp)

23. Benilov MS, Marotta A (1995) A model of the cathode region of atmospheric pressure arcs. J Phys D Appl Phys 28:1869-1882

24. Dabringhausen L, Nandelstädt D, Luhmann J, Mentel J (2002) Determination of HID electrode falls in a model lamp I: pyrometric measurements. J Phys D Appl Phys 35:1621-1630

25. Luhmann J, Lichtenberg S, Langenscheidt O, Benilov MS, Mentel J (2002) Determination of HID electrode falls in a model lamp II: Langluir-probe measurements. J Phys D Appl Phys 35:1631-1638 
26. Nandelstädt D, Redwitz M, Dabringhausen L, Luhmann J, Lichtenberg S, Mentel J (2002) Determination of HID electrode falls in a model lamp III: results and comparison with theory. J Phys D Appl Phys 35:1639-1647

27. Lichtenberg S, Nandelstädt D, Dabringhausen L, Redwitz M, Luhmann J, Mentel J (2002) Observation of different modes of cathodic arc attachment to HID electrodes in a model lamp. J Phys D Appl Phys 35:1648-1656

28. Dabringhausen L, Langenscheidt O, Lichtenberg S, Redwitz M, Mentel J (2005) Different modes of arc attachment at HID cathodes: simulation and comparison with measurements. J Phys D Appl Phys 38:3128-3142

29. Redwitz M, Langenscheidt O, Mentel J (2005) Spectroscopic investigation of the plasma boundary layer in front of HID-electrodes. J Phys D Appl Phys 38:3143-3154

30. Javidi Shirvan A (2013) Modelling of electric arc welding: arcelectrode coupling. Lic. Thesis. Chalmers University of Technology, Gothenburg

31. Schmitz H, Riemann KU (2001) Consistent analysis of the boundary layer of a Saha plasma. J Phys D Appl Phys 34:1193-1202

32. Riemann KU (1991) The Bohm criterion and sheath formation. J Phys D Appl Phys 24:494-518

33. Lee TH, Greenwood A, Breingan WD (1966) A self-consistent model for cathode region of a high pressure arc. Proc. 7th Int. Conf. on ionization phenomena in gases. Gradwenska Knjega, Beograd Yugoslavia, p 670

34. Morrow R, Lowke JJ (1993) A one-dimensional theory for the electrode sheaths of electric arcs. J Phys D Appl Phys 26:634-642

35. Zhu P, Lowke JJ, Morrow R, Haidar J (1995) Prediction of anode temperatures of free burning arcs. J Phys D Appl Phys 25:13691376

36. Lowke JJ, Morrow R, Haidar J (1997) A simplified unified theory of arcs and their electrodes. J Phys D Appl Phys 30:2033-2042

37. Sansonnens L, Haidar J, Lowke JJ (2000) Prediction of properties of free burning arcs including effects of ambipolar diffusion. J Phys D Appl Phys 33:148-157

38. Haidar J (1999) Non-equilibrium modelling of transferred arcs. J Phys D Appl Phys 32:263-272

39. Tanaka M, Teresaki H, Ushio M, Lowke J (2003) Numerical study of a free burning argon arc with anode melting. Plasma Chem Plasma Process 23:585-606

40. Ushio M, Tanaka M, Lowke JJ (2004) Anode melting from free burning argon arcs. IEEE Trans Plasma Sci 32:108-117

41. Lowke JJ, Tanaka M, Ushio M (2005) Mechanisms giving increased weld depth due to a flux. J Phys D Appl Phys 38:34383445

42. Füssel U, Lohse M, Rose S (2014) Sheath modelling of TIG welding. IIW Joint intermediate meeting Comm. IV, XII. SG212, Wels

43. Lowke JJ, Tanaka M (2006) 'LTE-diffusion approximation' for arc calculations. J Phys D Appl Phys 39:3634-3645

44. Murphy AB, Tanaka M, Tashiro S, Sato T, Lowke JJ (2009) A computational investigation of the effectiveness of different shielding gas mixtures for arc welding. J Phys D Appl Phys 42: 115205 (14pp)

45. Schnick M, Füssel U, Hertel M, Haessler M, Spille-Kohoff A, Murphy AB (2010) Modelling of gas-metal arc welding taking into account metal vapour. J Phys D Appl Phys 43:434008 (11pp)

46. Tanaka M, Yamamoto K, Tashiro S, Nakata K, Yamamoto E, Yamazaki K, Suzuki K, Murphy AB, Lowke JJ (2010) Timedependent calculations of molten pool formation and thermal plasma with metal vapour in gas tungsten arc welding. J Phys D Appl Phys 43:434009 (11pp)

47. Tang KM, Yan JD, Chapman C, Fang MT (2010) Threedimensional modelling of a dc arc plasma in a twin-torch system. J Phys D Appl Phys 43:345201 (15pp)
48. Murphy AB (2011) A self-consistent three-dimensional model of the arc, electrode and weld pool in gas-metal arc welding. J Phys D Appl Phys 44:194009 (11pp)

49. Ogino Y, Hirata Y, Nomura K (2011) Numerical analysis of the heat source characteristics of a two-electrode TIG arc. J Phys D Appl Phys 44:215202 (7pp)

50. Zhou X, Heberlein J, Pfender E (1994) Theoretical study of factors influencing arc erosion of cathode. IEEE Trans Compon Packag Manuf Technol Part A 17:107-112

51. Zhou X, Heberlein J (1994) Analysis of the arc-cathode interaction of free-burning arcs. Plasma Sources Sci Technol 3:564-574

52. Coulombe S (1997) A model of electric arc attachment on nonrefractory (cold) cathode, Ph.D. thesis. McGill University, Montréal

53. Coulombe S, Meunier J-L (1997) Arc-cold cathode interactions: parametric dependence on local pressure. Plasma Source Sci Technol 6:508-517

54. Cayla F, Freton P, Gonzalez J-J (2008) Arc/cathode interaction model. Plasma Sci IEEE Trans 36:1944-1954

55. Javidi Shirvan A, Choquet I, Nilsson H (2014) Modeling of electrode-arc coupling in electric arc welding. 14th Swedish Production Symposium SPS14, Gothenburg

56. Richley E, Tuma DT (1982) On the determination of particle concentrations in multi-temperature plasmas. J Appl Phys 53:85378542

57. Li HP, Pfender E, Chen X (2003) Application of Steenbeck's minimum principle for three-dimensional modelling of DC arc plasma torches. J Phys D Appl Phys 36:1084-1096

58. Benilov MS, Naidis GV (2010) What is the mathematical meaning of Steenbeck's principle of minimum power in gas discharge physics? J Phys D Appl Phys 43:175204 (2p)

59. Van de Sanden MCM, Schram P, Peeters AG, Van der Mullen JAM, Kroesen GMW (1989) Thermodynamic generalization of the Saha equation for a two-temperature plasma. Phys Rev A 40:5273-5276

60. Choquet I, Lucquin-Desreux B (2005) Hydrodynamic limit for an arc discharge at atmospheric pressure. J Stat Phys 41:197-239

61. Choquet I, Degond P, Lucquin-Desreux B (2007) A hierarchy of diffusion models for ionized plasmas. J Discrete Continuous Dyn Syst Series-B 8:735-772

62. Choquet I, Lucquin-Desreux B (2011) Non equilibrium ionization in magnetized two-temperature thermal plasma. Kinet Relat Models 4:669-700

63. Hsu KC, Pfender E (1983) Analysis of the cathode region of a freeburning high intensity argon arc. J Appl Phys 54:3818-3824

64. Simonin O, Delalondre C, Viollet P-L (1992) Modelling in thermal plasma and electric arc column. Pure Appl Chem 64:623-628

65. Benilov MS (2002) Theory and modelling of arc cathodes. Plasma Sources Sci Technol 11:A49

66. Benilov MS, Cunha MD, Naidis GV (2005) Modelling interaction of multi-species plasmas with thermionic cathodes. Plasma Sources Sci Technol 14:517-524

67. Benilov MS, Carpaij M, Cunha MD (2006) 3D modelling of heating of thermionic cathodes by high-pressure arc plasmas. $\mathrm{J}$ Phys D Appl Phys 39:2124-2134

68. Li HP, Benilov MS (2007) Effect of a near cathode sheath on heat transfer in high pressure arc plasmas. J Phys D Appl Phys 40:2010 2017

69. Benilov MS, Benilova LG, Li HP, Wu GW (2012) Sheath and arccolumn voltages in high-pressure arc discharges. J Phys D Appl Phys 45:355201 (10pp)

70. Baeva M, Uhrlandt D, Benilov MS, Cunha MD (2013) Comparing two non-equilibrium approaches to modelling of a free-burning arc. Plasma Sources Sci Technol 22:065017 (9pp)

71. Wendelstorf $\mathbf{J}(2000) \mathrm{Ab}$ initio modelling of thermal plasma gas discharges (electric arcs). Ph.D. thesis. Braunschweig University, Germany 
72. Rethfeld B, Wendelstrof J, Klein T, Simon G (1996) A selfconsistent model for the cathode fall region of an electric arc. $\mathrm{J}$ Phys D Appl Phys 29:121-128

73. Wendelstrof J, Decker I, Wohlfahrt H, Simon G (1997) TIG and plasma arc modelling: a survey. Mathematical Modelling of Weld Phenomena 3, The Institute of Materials, pp. 848-897.

74. Schmitz H, Riemann KU (2002) Analysis of the cathodic region of atmospheric pressure discharges. J Phys D Appl Phys 35:17271735

75. Baeva M, Kosakov R, Gorchakov S, Uhrlandt D (2012) Twotemperature chemically non-equilibrium modelling of transferred arcs. Plasma Sources Sci Technol 21:055027 (13pp)

76. Baeva M, Gorchakov S, Kosakov R, Uhrlandt D, Schoenemann T (2013) Non-equilibrium modelling of the electrical characteristics of a free-burning arc. High Voltage Eng 39:2159-2165

77. Gorchakov S, Baeva M, Kosakov R, Uhrlandt D, Schoenemann T (2014) Nonequilibrium arc model for the description of arcelectrode interaction. 27th Int. Conf. Electrical Contacts, Dresden, pp 534-539

78. Uhrlandt D, Baeva M, Pipa AV, Kozakov R, Gött G (2015) Cathode fall voltage of TIG arcs from a non-equilibrium arc model. Weld World 59:127-135

79. Rat V, Murphy AB, Aubreton J, Elchinger MF, Fauchais P (2008) Treatment of non-equilibrium phenomena in thermal plasma flows. J Phys D Appl Phys 41:183001 (28pp)

80. Potapov AV (1986) Chemical equilibrium of multi-temperature systems. High Temp 8:48-51

81. Choquet I (1995) Vibrational non-equilibrium modeling using direct simulation. Part 1: continuous internal energy. J Thermophys Heat Tran 9:446-455
82. Choquet I, Javidi Shirvan A, Nilsson H (2012) On the choice of electromagnetic model for short high-intensity arcs, applied to welding. J Phys D Appl Phys 45:205203 (14pp)

83. Haddad GN, Farmer AJD (1984) Temperature determinations in a free-burning arc. I. Experimental techniques and results in argon. J Phys D Appl Phys 17:1189-1196

84. Wienecke R (1955) Über das Geschwindigkeitsfeld der Hochstromkohlebogensäule. Zeitschrift für Physik 143:128-140

85. Haidar J, Farmer AJD (1991) A method for measurement of the cathode surface temperature for a high-current free-burning arc. Plasma Process 11:81-101

86. Haidar J, Farmer H (1995) Surface temperature measurements for tungsten-based cathodes of high-current free-burning arc. J Phys D Appl Phys 28:2494-2504

87. Hsu KC, Etemadi K, Pfender E (1983) Study of the free-burning high intensity argon arc. J Appl Phys 54:1293-1301

88. Coudert J-F, Grimaud A (1989) Étude expérimentale d'un générateur à arc transféré sous argon à la pression atmosphérique. Report EDF 44/89.31

89. Maecker H (1960) Messung und Auswertung von Bogencharakteristiken (Ar, N2). Zeitschrift für Physik 158:392404

90. Mitrofanov NK, Shkol'nik SM (2007) Two forms of attachment of an atmospheric-pressure direct-current arc in argon to a thermionic cathode. Tech Phys 52:711-720

91. Benilov MS (1999) Analysis of ionization non-equilibrium in the near-cathode region of atmospheric-pressure arcs. J Phys D Appl Phys 32:257-262

92. Hinnov E, Hirschberg JG (1962) Electron-ion recombination in dense plasmas. Phys Rev 125:795-801 